This is the author's copy of the publication as archived with the DLR's electronic library at http://elib.dlr.de . Please consult the original publication for citation.

\title{
Active Guidance of a Railway Running Gear with Independently Rotating Wheels
}

\author{
Andreas Heckmann, Alexander Keck; Gustav Grether
}

The development of railway running gears with independently rotating wheels is a focus of research within the Next Generation Train project at DLR. The lateral guidance of this type of running gears represents a challenging problem that relies on active control. The paper introduces results on this research regarding observer and control design that arefocused and demonstrated on a scaled experimental running gear hardware that was specifically constructed for this purpose. Additionally, the development of the lateral guidance system is supported by simulation and optimization within a Modelica based virtual simulation environment tailored for the modeling of railway vehicle dynamics in multi-domain engineering tasks.

\section{Copyright Notice}

(C2020 IEEE. Personal use of this material is permitted. Permission from IEEE must be obtained for all other uses, in any current or future media, including reprinting/republishing this material for advertising or promotional purposes, creating new collective works, for resale or redistribution to servers or lists, or reuse of any copyrighted component of this work in other works.

Heckmann, A., Keck, A., Grether, G. : Active Guidance of a Railway Running Gear with Independently Rotating Wheels, 2020 IEEE Vehicle Power and Propulsion Conference, VPPC 2020 - Proceedings 


\title{
Active Guidance of a Railway Running Gear with Independently Rotating Wheels
}

\author{
Andreas Heckmann, Alexander Keck, Gustav Grether \\ Institute of System Dynamics and Control \\ German Aerospace Center (DLR) \\ Wessling, Germany \\ andreas.heckmann@dlr.de
}

\begin{abstract}
The development of railway running gears with independently rotating wheels is a focus of research within the Next Generation Train project at DLR. The lateral guidance of this type of running gears represents a challenging problem that relies on active control. The paper introduces results on this research regarding observer and control design that are focused and demonstrated on a scaled experimental running gear hardware that was specifically constructed for this purpose. Additionally, the development of the lateral guidance system is supported by simulation and optimization within a Modelica based virtual simulation environment tailored for the modeling of railway vehicle dynamics in multi-domain engineering tasks.

Index Terms - railway running gear, observation and control of independently rotating wheels, Modelica
\end{abstract}

\section{INTRODUCTION}

The label "Next Generation Train" (NGT) entitles both, a long-term research project to which 11 DLR institutes contribute and a family of train concepts to consistently demonstrate the wide range of railway vehicle technologies under research at these institutes.

The major characteristics of the train design under investigation are very high speed up to $400 \mathrm{~km} / \mathrm{h}$ in daily operation, double deck configuration, light-weight design, improved passenger comfort, reduced life-cycle-costs and energy consumption per passenger. As regards running gears, wheel pairs with independently rotating and driven wheels (IRW) were chosen because of their promising but so far unexploited advantages. This design offers the capability of almost perfect steering along curves and facilitates continuous floors even on

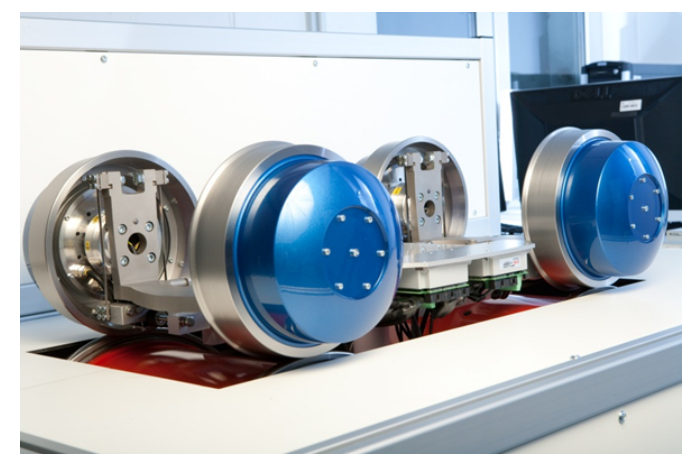

Fig. 1. Experimental IRW running gear on the 1:5 scale roller rig at DLR the lower level of the double deck car body, which would have to be stepped for a conventional wheel set axle. [1].

However, the task of guidance along the track or the lateral dynamics of the IRW, respectively, relies on active control in order to minimize wear and noise. And furthermore, it depends on sufficiently precise information on the lateral position of the wheel pair relative to the track, which is difficult to directly measure in daily train operation. Against this background, this paper reports on the nonlinear control and observer design for the IRW that is theoretically investigated, virtually simulated and experimentally demonstrated using the example of a 1:5 scale experimental running gear that was specifically constructed for this purpose, see Fig. 1.

The paper is organized as follows: Sec. II introduces the design of the scaled experimental running gear hardware, Sec. III is dedicated to the simulation environment, Sec. IV presents the observer design while Sec. V reports on the control synthesis. The final Sec. VI concludes the paper, refers to accompanying work on true scale simulation models and gives an outlook.

\section{Experimental RunNing Gear Hardware}

The major components of the experimental running gear in Fig. 2 are the central frame, the two axle bridges, and the four independently rotating wheels.

Each wheel is equipped with a permanent magnet synchronous motor as in-wheel drive, which is controlled by its own power converter. The actuating torques $\tau_{i}, i \in\{f, r\}$,

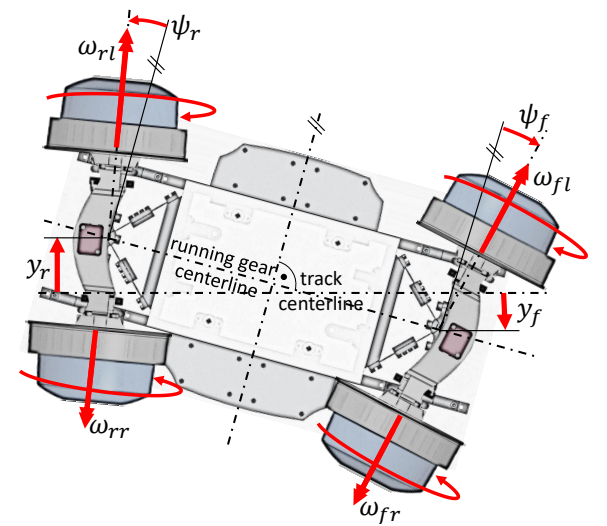

Fig. 2. Top view of the scaled experimental running gear 
where $f$ denotes front and $r$ rear, are applied to the wheels of each axle configuration in a differential manner, i.e. $\tau_{i j}= \pm \tau_{i}$, $j \in\{r, l\}$, where $r$ signifies right and $l$ left.

The axle bridges are connected to the running gear frame via a leaf spring guidance. The mounting and the vertical spring represent the primary suspension of the running gear. This configuration enables a yaw motion of the axle bridges, that is limited by bump stops. The central frame is attached to the roller rig with a lemniscate guidance that locks the longitudinal motion.

Fig. 2 displays the degrees of freedom of the running gear. These are the four wheel rotations with speeds $\omega_{i j}$, the yaw angles of the axle-bridges $\psi_{i}$ and the lateral displacements of the axle bridge centers $y_{i}, i \in\{f, r\}$, with respect to the track centerline. For detailed description see [2], where the equations of motion are given as well.

The sensor equipment of the experimental running gear is set-up to examine and compare different observer configurations. It includes four rotary encoders at each wheel in order to monitor $\omega_{i j}$. At each axle bridge, an angle sensor measures the yaw angle $\psi_{i}$ and an inertial measurement unit (IMU) i.a. provides information on $\ddot{y}_{i}$ and $\dot{\psi}_{i}$. The signals of four force-torque sensors at the attachment points of each wheel drive to the axle bridges are evaluated in such a way that the resultant lateral force $F_{y, i}$, the resultant vertical force $F_{z, i}$ and the resultant torque $M_{x, i}$ around the longitudinal axis for each axle bridge are available and signal drift is canceled out.

In order to provide reference signals, laser distance sensors between roller rig base and central frame are mounted to measure $y_{i}$ in addition. Under real life conditions at railway tracks, these lateral positions are difficult to measure in a reliable and robust manner, which motivates the design of appropriate observers.

The rapid control prototyping system of the roller rig facilitates processing of all sensor and actuator signals within observer and control algorithms in real-time.

\section{Simulation ENVIRONMENT}

Modeling, simulation and optimization are important tools besides hardware testing that offer the opportunity to examine and evolve new technical concepts in early design phases without implementation risks and at comparabely low costs.

An attractive approach in the context of control development and multidomain simulation is the object-oriented modelling language Modelica that is an open standard developed by the non-profit Modelica Association [3]. Compared to blockoriented general environments, Modelica models are built up on mathematical equations that are symbolically pre-processed and do not rely on an a priori known signal flow [4]. Since the symbolic pre-proccessing may also include the inversion of nonlinear plant models, Modelica is in particular helpful to systematically design model-based controllers for nonlinear systems [5].

Moreover, Modelica supports an all-in-one-tool-approach, i.e. it provides the capability to cover multi-domain engineering tasks in one consistent simulation environment and is in particular tailored to represent of the dynamic behavior of heterogeneous physical systems including e.g. mechanical, pneumatic, electric or hydraulic components.

As regards railway vehicle systems, the DLR Railway Dynamics Library [6] shown in Fig. 3 has recently been released

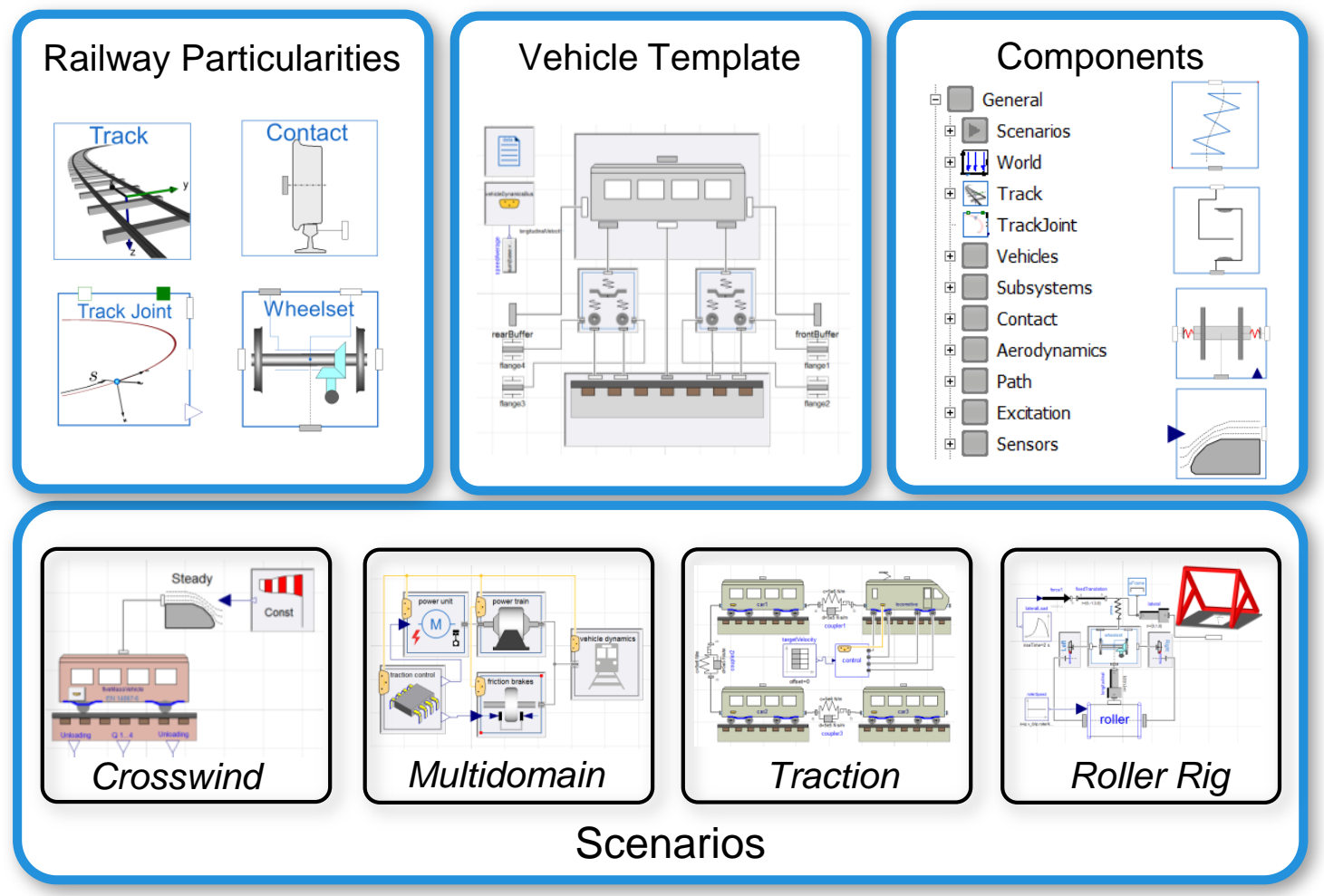

Fig. 3. Overview on major items of the Modelica Railway Dynamics Library. 


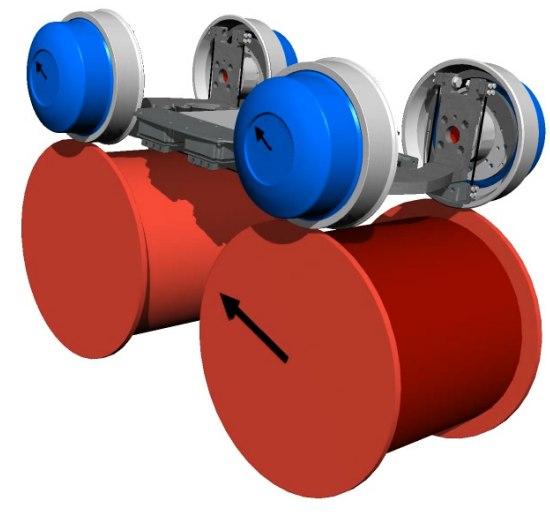

Fig. 4. Visualization of the experimental running gear model

and extends the wide range of domain specific component libraries that are publicly available either on a open source or a commercial basis. It supports the consideration of vehicle dynamics issues such as traction, comfort and safety in multidomain engineering tasks. Vehicle, track, wheel-rail contact models and roller rig scenarios are prepared on different levels of detail and offer interfaces to subsystem models that represent e.g. electric propulsion engines, pneumatic brakes or control algorithms. Fig. 4 presents the visualization of the simulation model used to develop and optimize the observation and control algorithms for the experimental running gear at DLR.

\section{OBSERVER DESIGN}

Consider the unknown real life system

$$
\dot{\boldsymbol{x}}=\boldsymbol{f}\left(\boldsymbol{x}, u, v_{R}\right)+\boldsymbol{\mu}, \quad \boldsymbol{y}=\boldsymbol{g}\left(\boldsymbol{x}, u, v_{R}\right)+\boldsymbol{\rho}
$$

of a single axle configuration, on which the discussion is focused from now on, since observation and control of each axle is treated independently. The states and the outputs of the system are represented by $\boldsymbol{x}$ and $\boldsymbol{y}$, while $u$ denotes the system input and $v_{R}$ the given longitudinal speed. The process and measurement noise contributions $\boldsymbol{\mu}$ and $\rho$ are presumed to be additive and Gaussian with zero mean.

The equations of motion from [2] are reformulated and extended to specify observer equations of the following form:

$$
\dot{\hat{\boldsymbol{x}}}=\hat{\boldsymbol{f}}\left(\hat{\boldsymbol{x}}, u, v_{R}\right)+\boldsymbol{L}\left(\hat{\boldsymbol{x}}, u, v_{R}\right)(\boldsymbol{y}-\hat{\boldsymbol{y}}), \quad \hat{\boldsymbol{y}}=\hat{\boldsymbol{g}}\left(\hat{\boldsymbol{x}}, u, v_{R}\right) .
$$

Here, $\hat{x}$ and $\hat{\boldsymbol{y}}$ symbolize the estimates of the associated quantities in (1), while $\boldsymbol{L}(\hat{\boldsymbol{x}}, u, v)$ denotes the so far unkown rule to feed back the output error constituted by comparison of the observer outputs $\hat{\boldsymbol{y}}$ and the measurements of the real life system $\boldsymbol{y}$.

Two options appear: The more elaborate initial system assumes the state vector $\hat{\boldsymbol{x}}_{6}$

$$
\hat{\boldsymbol{x}}_{6}=\left[y_{i}, \dot{y}_{i}, \psi_{i}, \dot{\psi}_{i}, \omega_{i r}, \omega_{i l}\right]^{T},
$$

while the reduced state vector $\hat{\boldsymbol{x}}_{5}$ could be defined neglecting the lateral wheel slip [7]:

$$
\dot{y}_{i} \sim v \psi_{i} \Longrightarrow \hat{x}_{5}=\left[y_{i}, \psi_{i}, \dot{\psi}_{i}, \omega_{i r}, \omega_{i l}\right]^{T} .
$$

\begin{tabular}{|c|c|}
\hline configurations & $\Delta y_{i}$ \\
\hline$\hat{\boldsymbol{x}}_{5}, \quad \hat{\boldsymbol{g}}_{3}$ & $\approx 5 \cdot 10^{-4} \mathrm{~m}$ \\
\hline$\hat{\boldsymbol{x}}_{6}, \quad \hat{\boldsymbol{g}}_{5}$ & $\approx 1.4 \cdot 10^{-4} \mathrm{~m}$ \\
\hline$\hat{\boldsymbol{x}}_{6}, \quad \hat{\boldsymbol{g}}_{6}$ & $\approx 1.2 \cdot 10^{-4} \mathrm{~m}$ \\
\hline
\end{tabular}

TABLE I

EXPECTED ERROR DUE TO ERROR PROPAGATION OF SENSOR ACCURACIES

It is an important objective of research to examine several alternative sensor configurations and to tell their properties. That is why the following three sensor compositions are taken under consideration:

$$
\begin{aligned}
& \hat{\boldsymbol{g}}_{3}=\left[\psi_{i}, \omega_{i r}, \omega_{i l}\right]^{T}, \\
& \hat{\boldsymbol{g}}_{5}=\left[\psi_{i}, \omega_{i r}, \omega_{i l}, \ddot{y}_{i}, \dot{\psi}_{i}\right]^{T}, \\
& \hat{\boldsymbol{g}}_{6}=\left[\psi_{i}, \omega_{i r}, \omega_{i l}, F_{y, i}, F_{z, i}, M_{x, i}\right]^{T} .
\end{aligned}
$$

A rigorous nonlinear observability analysis proved that observability is given for both, the reduced and the full system for all sensor configurations as long as $v_{R} \neq 0$. However, the low level configuration $\hat{\boldsymbol{g}}_{3}$ might not be precise enough during acceleration or deceleration processes with high slip conditions.

Eqs. (5) to (7) may be used to analyze the expected estimation error $\Delta y_{i}$, which follows from the specified sensor errors and their propagation through the measurement equations. The results in Table I present the advantage of the high level configurations $\hat{\boldsymbol{g}}_{5}$ and $\hat{\boldsymbol{g}}_{6}$ with respect to the estimation accuracy.

Following the idea of the Kalman Filter, $\boldsymbol{\mu}$ and $\rho$ from (1) or the associated covariance matrices $\boldsymbol{Q}$ and $R$, respectively, could be used to substantiate the rule $\boldsymbol{L}\left(\hat{\boldsymbol{x}}, u, v_{R}\right)$ for (2). For nonlinear systems, several algorithms such as the Extended (EKF) or the Unscented Kalman Filter (UKF) exist that provide estimates of the current state variables along with their uncertainties, cf. [8].

With this background, the specific synthesis task consists of the design of $Q$ and $R$ in such a way that the convergence of the estimation error is ensured. Provided that real life measurement data $\boldsymbol{y}$ are available, numerical optimization of the following problem with multiple cases $S_{k} \in S$ is an appropriate tool to do so [9]:

$$
\begin{gathered}
\min _{\nu} \max _{S_{k} \in S} o_{k}(\nu), \quad \nu:=([\operatorname{diag}(\boldsymbol{Q}), \operatorname{diag}(\boldsymbol{R})]), \\
o_{k}:=\frac{1}{t_{k}} \int_{0}^{t_{k}}|\boldsymbol{y}-\hat{\boldsymbol{y}}| \mathrm{d} t .
\end{gathered}
$$

Hereby, the set $S$ is supposed to cover all relevant application scenarios and to truely represent the circumstances in daily operations including e.g. disturbances, parameter variations, switching operations and so on. The specific formulation of the optimization as a minimization of the worst case in (8) as well supports the aim to get robust observer parametrization.

In order to define a demanding scenario and demonstrate the accuracy and robustness of the observer, fluctuating running velocities according to Fig. 5 are specified. Fig. 6 shows 


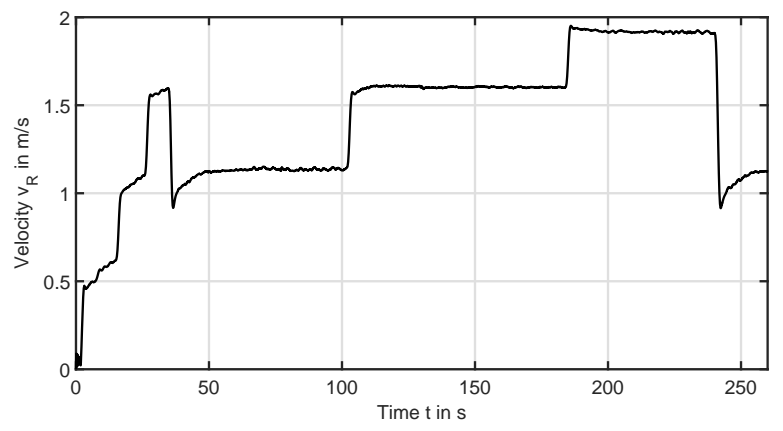

Fig. 5. Velocity profile of the scenario.

selected observation results for the low-level configuration $\hat{\boldsymbol{g}}_{3}$ using the EKF algorithm. Since additional reference sensors to measure $y_{i}$ are available at the test rig, the estimation error $\left(y_{i}-\hat{y}_{i}\right) / y_{i}$ could be plotted in Fig. 7 . Its values are in the expected order of magnitude, since they correspond to the estimations given in Table I.

\section{Control Synthesis}

IRW are not prone to show the hunting motion and the associated instability that is well known from classical wheelset design. However as the other side of the medallion, they do not provide sufficient guidance characteristics or more precise: In unassisted, free motion, the lateral displacements $y_{i}$ tend to increase till flange contact with high wear occur. This is why guidance is to be supplied by active control here.

The concept of feedback linearization in combination with model inversion [5] is applied in order to introduce the significant influence of the running velocity $v$ on the system dynamics, but to avoid gain scheduling between linear designs at various operating points.

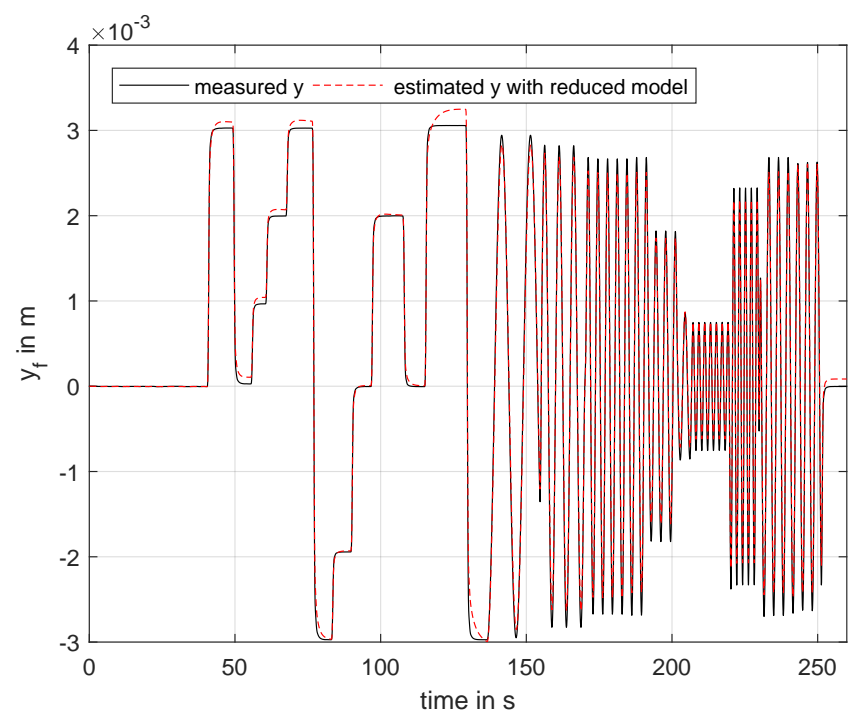

Fig. 6. EKF estimation results of the $\hat{\boldsymbol{g}}_{3}$ observer setting compared with reference measurements.

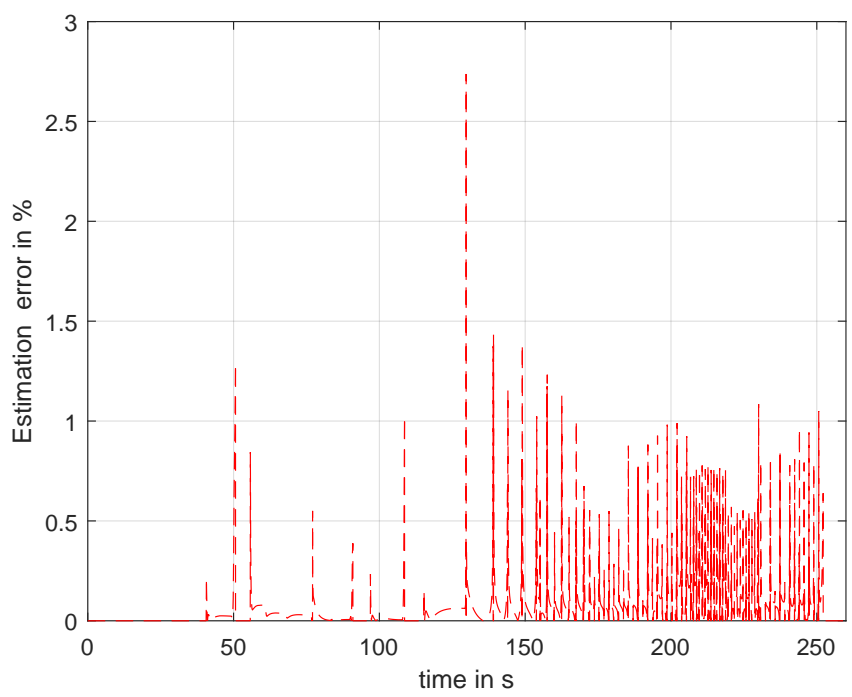

Fig. 7. EKF estimation errors for the $\hat{\boldsymbol{g}}_{3}$ observer setting.

It turned out that, compared to Sec. IV, the design model for control synthesis could be further simplified neglecting all creepage terms. With respect to the lateral dynamics this corresponds to the reduction in (4). If in addition ideal rolling of the wheels is assumed, the rotational velocities $\omega_{i j}$ could be eliminated from the equations and the minimal state vector $\hat{\boldsymbol{x}}_{3}=\left(y_{i}, \psi_{i}, \dot{\psi}_{i}\right)^{T}$ remains.

Furthermore, the cascaded control structure in Fig. 8 is proposed for the experimental running gear assuming sufficiently differentiable set point signals. The corresponding Eq. (9) bases on the constraint equation in (4) with proportionality constant $\xi \approx 1$ that is associated to the wheel-rail conicity, cf. (4) in [7]. Eq. (10) originates from the equation of motion with respect to yaw. Set point or desired values, respectively, are indicated by a superscripted asterisk, i.e. (... ${ }^{*}, J$ denotes the moment of inertia of the axle bridge configuration, $2 b$ the track gauge and $r$ the nominal wheel radius:

$$
\begin{array}{ll}
f_{1}^{-1}: & {\left[\left(y_{i}^{*}-y_{i}\right) k_{y}+\dot{y}_{i}^{*}\right] \frac{1}{\xi v_{R}}=\psi_{i}^{*}} \\
f_{2}^{-1}: & {\left[J \ddot{\psi}_{i}^{*}+\left(\dot{\psi}_{i}^{*}-\dot{\psi}_{i}\right) k_{\dot{\psi}}+\left(\psi_{i}^{*}-\psi_{i}\right) k_{\psi}\right] \frac{r}{2 b}=\tau_{i}}
\end{array}
$$

Note that these two equations are prepared to consider nonlinearities e.g. with respect to the wheel-rail profile geometry

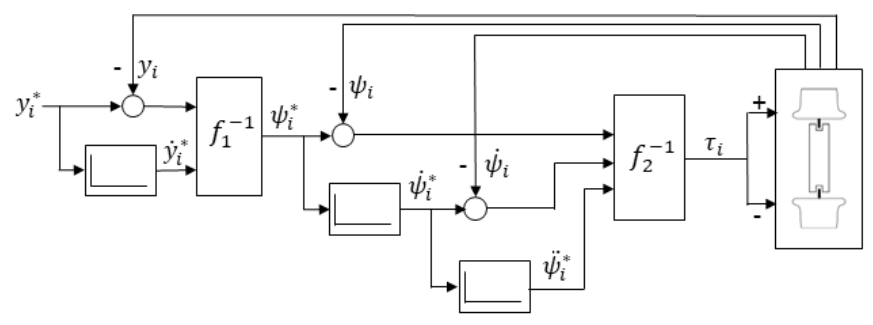

Fig. 8. Cascaded control structure for the M 1:5 experimental running gear. 


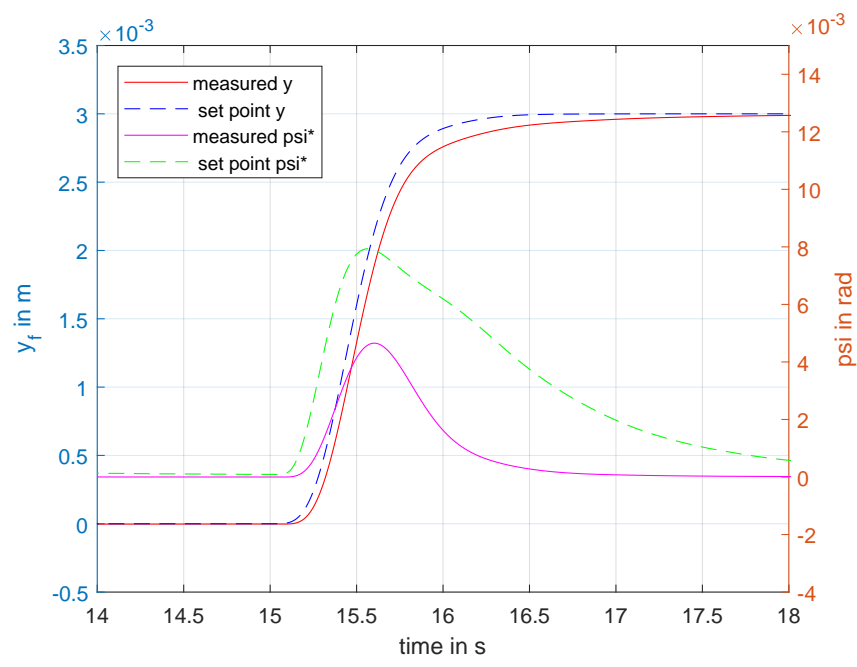

Fig. 9. Control optimization scenario

by specifying $\xi=\xi(y, \psi)$ and $r=r(y, \psi)$. Related benefits and robustness issues are subjects of current research.

The three feedback gains $\mu:=\left(k_{y}, k_{\psi}, k_{\dot{\psi}}\right)$ in (9) and (10) were specified by optimization. To this end, we define the set point trajectory in Fig. 9, which is a lateral displacement of $3 \mathrm{~mm}$ within $2.5 \mathrm{~s}$, but low-pass filtered with cut-off frequency of $3 \mathrm{~Hz}$. A constant speed $v=1 \mathrm{~m} / \mathrm{s}$ is assumed.

The velocity profile in Fig. 5 was applied to demonstrate the implementation of the control at the experimental running gear hardware in Fig. 10.

It is obvious that the IRW running gear is capable of following an almost arbitrary trajectory mainly limited by the given track clearance and the available actuation power. The general objective of IRW control is to limit $y_{i}$ within a specific range, where no flange contacts occur, so that wear and noise emmissions are reduced. Active steering into curves with transitions and not only reducing but evenly distributing wear by defining appropriate trajectories $y_{i}^{*}$ are further objectives.

However, real railway tracks are technical systems, where rail irregularities permanently excite the lateral motion of the running gears. So the roller rig scenario here is an idealized one and steady state accuracy is actually not a control design goal in practise.

\section{Conclusions AND OUTLOOK}

The paper presents the development of an observer and control design for railway running gears with independently rotating wheels. Hereby, the presentation is focused on the M1:5 hardware constructed at DLR in order to provide an experimental environment. The research is additionally supported by virtual simulations and optimization within the Modelica based Railway Dynamics Library. The transfer of the results to true scale systems is ongoing work and already prepared in M1:1 multibody simulations [10]. To go one step further, an experimental running gear in true scale is currently beeing

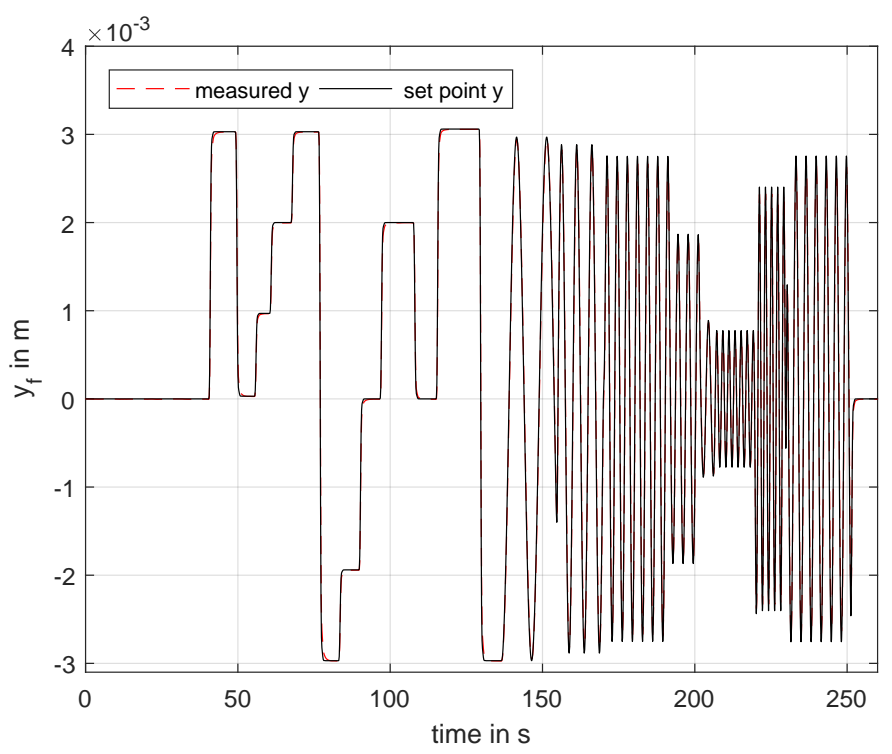

Fig. 10. Comparison of the set point and the measured trajectory

constructed at DLR. It is expected to be available for first experiments in the course of the year 2021 .

\section{REFERENCES}

[1] J. Winter, E. Mittelbach, and J. Schykowski, Eds., RTR Special - Next Generation Train. Eurailpress, DVV Media Group, 2011.

[2] C. Schwarz, A. Heckmann, and A. Keck, "Different models of a scaled experimental running gear for the DLR RailwayDynamics Library," in 11th International Modelica Conference, 21.-23. Sep. 2015, 2015.

[3] Modelica, "Modelica ${ }^{\circledR}$ - a unified object-oriented language for physical systems modeling - language specification 3.4," 2017, https://www.modelica.org/documents/ModelicaSpec34.pdf.

[4] M. Otter, H. Elmqvist, and S. Mattsson, "Multi-domain Modeling with Modelica," in CRC Handbook of Dynamic System Modeling, P. Fishwick, Ed. Chapman \& Hall, 2007, pp. 36.1 - 36.27.

[5] M. Thümmel, G. Looye, M. Kurze, M. Otter, and J. Bals, "Nonlinear inverse models for control," in Proceedings of the 4th International Modelica Conference, 2005, pp. 267-279.

[6] A. Heckmann, M. Ehret, G. Grether, A. Keck, D. Lüdicke, and C. Schwarz, "Overview of the DLR RailwayDynamics Library," in 13th International Modelica Conference, 2019.

[7] A. Keck, C. Schwarz, and T. Meurer, "Observer design for a railway running gear with independently rotating wheels," in Joint 8th IFAC Symposium on Mechatronic Systems and 11th IFAC Symposium on Nonlinear Control Systems, 2019. [Online]. Available: https://elib.dlr.de/127878/

[8] J. Brembeck, "Nonlinear constrained moving horizon estimation applied to vehicle position estimation," Sensors, vol. 19, no. 10, p. 2276, 2019. [Online]. Available: https://elib.dlr.de/127735/

[9] A. Pfeiffer, "Optimization library for interactive multi-criteria optimization tasks," in 9th International Modelica Conference, 2012.

[10] G. Grether, A. Heckmann, and G. Looye, "Lateral guidance control using information of preceding wheel pairs," in Advances in Dynamics of Vehicles on Roads and Tracks, IAVSD2019. Lecture Notes in Mechanical Engineering, M. Klomp, F. Bruzelius, J. Nielsen, and A. Hillemyr, Eds. Springer, 2020, pp. 863-871. 